\section{Ärztenachwuchs hat wenig Interesse an Chefarzt-Posten oder eigener Praxis}

Immer weniger Ärzte und vor allem Ärztinnen in Deutschland können sich eine freiberufliche Tätigkeit in einer eigenen Praxis vorstellen. Die meisten Nachwuchsärzte und -ärztinnen, die sich im vierten Jahr ihrer Weiterbildung befinden, möchten auch später im Krankenhaus bleiben oder als Facharzt angestellt sein. Die jüngsten Ergebnisse einer Längsschnittstudie in der Fachzeitschrift „DMW Deutsche Medizinische Wochenschrift" (DMW 2017; 142; e74e82) zeigen auch, dass viele Frauen keine Chefarzt-Karriere anstreben.

Die KarMed-Studie des Instituts für Allgemeinmedizin in Hamburg erforscht die Karrierewünsche von Nachwuchsärzten in Deutschland. Bereits erste Befragungen aus den Jahren 2008/2009 - die Mediziner befanden sich damals am Ende ihres Studiums im sogenannten Praktischen Jahr - zeigten die zukünftigen Ärzte wenig Interesse an traditionellen Berufskarrieren. Chefarzt einer großen Klinik oder Besitzer einer eigenen Praxis zu sein, war für viele kein erstrebenswertes Ziel. Vor allem Frauen, heute die Mehrzahl der Medizinstudierenden, gaben an, sie würden eine angestellte Tätigkeit vorziehen, gerne auch in Teilzeit.

Inzwischen befinden sich die Befragten im vierten Jahr ihrer beruflichen Weiterbildung, die in der Regel im Krankenhaus erfolgt. Die meisten leben in einer festen Partnerschaft. Etwa 30 Prozent haben Kinder. Obwohl das Ende der Weiterbildung bevorsteht und die Ärzte und Ärztinnen dann die Möglichkeit hätten, sich als Facharzt niederzulassen, möchten 51 Prozent weiter in der Klinik arbeiten. Und unter denjenigen, die gerne im ambulanten Bereich arbeiten möchten, würden viele eine angestellte Tätigkeit, etwa in einem medizinischen Versorgungszentrum, einer eigenen Praxis vorziehen. Bei den Frauen war dies mit 52 Prozent sogar die Mehrheit. Die meisten Ärztinnen würden gerne in Teilzeit arbeiten. Dies gilt insbesondere für jene, die Kinder haben.

Dass Mediziner das Krankenhaus als Arbeitsstätte bevorzugen, stellt rückblickend die wichtigste Veränderung der Berufspräferenzen seit Gründung der Bundesrepublik Deutschland dar, schreibt Stine Ziegler vom Institut für Allgemeinmedizin, Universitätsklinikum Hamburg-Eppendorf. Sie hat gemeinsam mit Hendrik van den Bussche und weiteren Kollegen die jüngste Umfrage ausgewertet. In der Vergangenheit habe die Mehrheit der Ärzte nach dem Ende der Weiterbildung eine selbstständige Tätigkeit angestrebt.

Am ehesten könnten sich Kinderärzte eine eigene Praxis vorstellen. Die Hälfte der männlichen und zwei Drittel der weiblichen Kinderärzte gaben an, dass sie eine Tätigkeit als Kassenarzt anstreben. Auch bei den Internisten, Orthopäden und Unfallchirurgen möchte die Mehrheit das Krankenhaus nach dem Ende der Weiterbildung verlassen. Für Anästhesisten kommt dies nur in seltenen Fällen infrage. Auch Chirurgen und Frauenärzte möchten mehrheitlich im Krankenhaus bleiben.

Dort streben nicht alle Ärzte eine Führungsposition an. Vor allem Ärztinnen würden sich häufig mit einer Tätigkeit als Assistenzarzt begnügen, berichtet Ziegler. Nur zwei Prozent der Medizinerinnen, die nach der fachärztlichen Anerkennung in der Klinik weiterarbeiten wollten, strebten eine Position als Chefärztin an. Auch der Oberarzt-Posten ist für Ärzte reizvoller als für ihre weiblichen Kolleginnen. Insgesamt war der Anteil der Ärztinnen, die „nur“ als Fachärztin ohne Leitungsaufgaben im Krankenhaus tätig bleiben wollten, dreimal größer als bei ihren männlichen Kollegen, und das, obwohl die Frauen unter den Nachwuchsärzten in der Mehrheit sind: Mehr als 60 Prozent aller Absolventen des Medizinstudiums sind weiblich.

Die Ergebnisse stimmen mit anderen Untersuchungen, etwa des Marburger Bundes, überein. Nach Ansicht von Ziegler und van den Bussche ist die Politik und hier vor allem die Kassenärztliche Bundesvereinigung gefragt. Diese müssten dem Nachwuchs neue Tätigkeitsformen anbieten, um sie für den ambulanten Bereich zu gewinnen. Ansonsten würden die Ärzte in andere Bereiche abwandern.

Nach einer Pressemitteilung

Georg Thieme Verlag 